\title{
UM MÉTODO VERDE, RÁPIDO E SIMPLES PARA DETERMINAR O VALOR ENERGÉTICO DE FARINHAS E CEREAIS MATINAIS
}

\author{
Juliana Terra, Alexandre Martinez Antunes e Maria Izabel Maretti Silveira Bueno* \\ Departamento de Química Analítica, Instituto de Química, Universidade Estadual de Campinas, CP 6154, 13084-971 Campinas - SP, Brasil \\ Marcelo Alexandre Prado \\ Departamento de Ciência de Alimentos, Faculdade de Engenharia de Alimentos, Universidade Estadual de Campinas, 13084-971 \\ Campinas - SP, Brasil
}

Recebido em 25/8/09; aceito em 13/1/10; publicado na web em 23/4/10

\begin{abstract}
A CLEAN, FAST AND SIMPLE METHOD TO DETERMINE THE ENERGY VALUE OF DRIED FOODS AND BREAKFAST CEREAL. The Energy Value (EV) corresponds to the sum of the energetic contributions from food macronutrients (proteins, carbohydrates and fats) and is required on the labels of pre-packaged foods. The determinations of these parameters are based on distinct analytical procedures, each one being time-consuming, laborious and producing residues. This work presents multivariate models to determine the EV contents of industrialized foods for human consumption by using X-ray fluorescence spectra of samples with known parameters, determined through conventional methods. The proposed method is an alternative to conventional analytical methods and does not require any reagent, given the demands of the "green chemistry".
\end{abstract}

Keywords: energy value; green chemistry; X-ray fluorescence.

\section{INTRODUÇÃO}

A dieta adequada de um adulto, segundo as recomendações internacionais, deve envolver um consumo diário de $2000 \mathrm{kcal}$. Para que a dieta seja caloricamente balanceada, 45 a 55\% deste total devem ser provenientes de carboidratos; 12 a $13 \%$ de proteínas; $10 \%$ de açúcares e 20 a $30 \%$ de gorduras. ${ }^{1}$

A energia que um alimento fornece ao organismo para que ele possa realizar suas funções biológicas é chamada de valor energético (VE). ${ }^{2}$ Nos países em que existem legislações sobre a rotulagem dos alimentos, informações sobre VE devem ser apresentadas obrigatoriamente em todos os produtos industrializados. ${ }^{2,3}$

Através do rótulo, o consumidor adquire dados para escolher os alimentos a fim de balancear sua dieta, evitando excessos e deficiências que possam acarretar problemas de saúde., ${ }^{4,5}$

Bryant e Atwater estudaram o comportamento dos alimentos verificando a disponibilidade de seus macronutrientes, ou seja, o quanto é liberado de calor na queima (oxidação) destes nutrientes. ${ }^{6}$ Os resultados permitiram constatar que, em média, cada grama de proteína ou de carboidrato libera $4 \mathrm{kcal}$ de energia, enquanto para cada grama de gordura este valor é $9 \mathrm{kcal} .{ }^{6}$ Desta forma, para obter VE pela relação estabelecida por Bryant e Atwater (Equação 1) é preciso obter as quantidades, em gramas, de carboidrato $(\mathrm{C})$, proteína $(\mathrm{P})$ e gordura $(\mathrm{G})$ presentes em cada grama de amostra $(\mathrm{g} / \mathrm{g}){ }^{7}$

$$
\mathrm{VE}=4 \mathrm{C}+4 \mathrm{P}+9 \mathrm{G}
$$

Os valores obtidos por Bryant e Atwater com relação à disponibilidade de seus macronutrientes foram resultados de estudos feitos a partir da ingestão mista (variada) de alimentos e por isso são conhecidos como fatores gerais de Atwater. ${ }^{7}$ Estudos têm sido feitos para verificar os valores específicos para cada tipo de alimento, constando pequenas variações nos fatores gerais e levando a constantes adaptações da Equação 1. Mesmo assim, a equação, tal como sugerida inicialmente, é aplicada e aceita pelos métodos oficiais atualmente. ${ }^{7}$

\footnotetext{
*e-mail: bell@iqm.unicamp.br
}

A determinação dos macronutrientes é feita a partir de procedimentos analíticos distintos, o que tende a aumentar os erros envolvidos no cálculo de VE pela equação de Bryant e Atwater, pois maiores são as possibilidades de problemas experimentais.

A Association of Official Analytical Chemists recomenda que a determinação do teor proteico de um alimento seja feita através da determinação do nitrogênio pelo método de Kjeldahl. ${ }^{8}$ Este método envolve três etapas (digestão, destilação e titulação da amostra), as quais requerem o uso de ácido sulfúrico e hidróxido de sódio concentrados, além de outros reagentes tóxicos.

Vários são os métodos encontrados na literatura para determinar a quantidade de lipídios nas amostras. ${ }^{9-13}$ Entre estes métodos, o Bligh-Dyer ${ }^{9}$ é o mais simples, pois não requer equipamentos sofisticados ou caros. Porém, para a sua execução, são necessárias grandes quantidades de clorofórmio, metanol e água para promover a extração da gordura da amostra.

O teor de carboidrato pode ser calculado pela diferença entre 100 e a soma das porcentagens de água, proteína, gorduras, álcool (quando presente) e cinzas. Portanto, no cálculo de carboidrato também são requeridos outros parâmetros, necessitando realizar diferentes e variados procedimentos para a obtenção destes. ${ }^{2}$

Todos os procedimentos descritos anteriormente são laboriosos, lentos, requerem o uso de reagentes tóxicos e geram muitos resíduos. Estes aspectos não são convenientes para análise de rotina e vão contra os princípios da chamada "química verde"., 7,14

Visando minimizar estes aspectos negativos foi desenvolvido um método, a partir da aliança entre a fluorescência de raios $\mathrm{X}$ (FRX) e a quimiometria, para determinação de VE de amostras de farinhas e alimentos matinais. Para ser aplicado, é necessária a obtenção de uma equação de calibração. Esta equação é específica para um tipo ou classe de produtos, no caso, farinhas e alimentos matinais. Esta aplicação não é genérica como os métodos analíticos convencionais. Dada a necessidade de um grande conjunto de dados experimentais para o ajuste e a validação, a relação custo-benefício deve ser avaliada para a substituição dos métodos convencionais pelo FRX. Com o modelo pronto, o benefício é, sem dúvida, muito grande. 
Os alimentos derivados de cereais contribuem em grande parte para a ingestão calórica da população em geral, uma vez que são consumidos em todo o mundo, por todas as faixas etárias, além de serem frequentemente usados como matéria-prima para a indústria alimentícia.

As farinhas são obtidas a partir da moagem dos cereais e são consumidas por grande parte da população, sendo uma das principais fontes de alimentos para a população de baixa renda. Paralelamente, os alimentos matinais são derivados de produtos extrusados de cereais que, em geral, se apresentam na forma de flocos, tais como os chamados flakes e as granolas. $\mathrm{O}$ consumo de cereais matinais tem aumentado muito nos últimos anos devido à necessidade de se obter produtos de preparo rápido, tendo em vista a falta de tempo da vida moderna. ${ }^{15}$

A FRX é uma técnica de análise relacionada com a medida de energia e intensidade características da radiação X emitida por uma amostra irradiada com radiação eletromagnética. ${ }^{16}$ Para os elementos ditos pesados $(Z>11)$, as informações das amostras por FRX são baseadas no efeito fotoelétrico, ou seja, na energia liberada no deslocamento eletrônico dos átomos da amostra quando esta é irradiada pela radiação X. Como cada elemento libera uma determinada energia neste processo, podem-se obter informações qualitativas da amostra. Paralelamente, a intensidade da energia emitida é proporcional à quantidade do elemento na amostra. ${ }^{17,18}$

Embora ainda seja uma aplicação recente, a FRX também fornece informações dos elementos leves das amostras, geralmente associados à parte orgânica das mesmas. Nestes casos, são as regiões de espalhamento do feixe incidente que conservam a informação. Como as variações destas regiões são muito tênues de amostra para amostra, a exploração das mesmas requer o auxílio de ferramentas quimiométricas..$^{19,20}$

A quimiometria é a área da química que emprega métodos matemáticos e estatísticos para planejar ou selecionar experimentos de forma otimizada, fornecendo o máximo de informações químicas com a análise dos dados. ${ }^{1}$

As principais ferramentas quimiométricas utilizadas são a análise por componentes principais (PCA - Principal Component Analysis) e a regressão por quadrados mínimos parciais, o PLS (Partial Least Squares). Ambas consistem na redução da dimensionalidade do conjunto de dados a partir de combinações lineares das variáveis originais, diferindo no tipo de dados que trazem da amostra: a PCA fornece informações qualitativas, permitindo reduzir a dimensionalidade dos dados, verificar a existência de amostras anômalas e as de maior importância, eliminar ruídos experimentais, classificar as amostras e/ou agrupá-las conforme suas semelhanças, etc. Já o PLS estabelece uma relação quantitativa entre o conjunto das respostas instrumentais e uma ou mais propriedades físicas ou químicas das amostras de interesse, desenvolvendo um modelo matemático que correlaciona estas informações. ${ }^{22-24}$

O método proposto não gera resíduos e não necessita de qualquer tipo de solvente ou reagente, atendendo às exigências da chamada "química verde". Além disso, a sua aplicação requer 0,2\% do tempo necessário para a aplicação dos métodos convencionais.

Os resultados obtidos pelo método proposto foram validados a partir da comparação com os obtidos pelos métodos convencionais e através de figuras de mérito calculadas conforme sugerido na literatura. ${ }^{25}$

\section{PARTE EXPERIMENTAL}

\section{Materiais}

Foram utilizadas 100 amostras comerciais de farinhas e cereais matinais de marcas variadas, obtidas em supermercados da região de Campinas/SP.
Todas as amostras eram derivadas de pelo menos um dos seguintes cereais: trigo, milho, soja, arroz, centeio, cevada e mandioca. Além do cereal presente, as amostras diferenciavam em outras características (presença de chocolate, açúcar, leite, frutas cristalizadas, etc) de maneira a se ter um grupo amostral bem diversificado.

As amostras foram trituradas com um moinho do tipo "martelo" (Tecnal, TE 600), com saída de abertura de 100 mesh, antes de serem utilizadas.

Todos os procedimentos realizados (convencionais e proposto) foram feitos em triplicata por amostra. As replicatas foram comparadas utilizando-se o teste $Q$, para verificar se diferiram significativamente entre si com $95 \%$ de confiança. ${ }^{26,27}$ Foi calculado o valor médio de cada parâmetro entre as replicatas não excluídas.

\section{Métodos}

Determinação do valor energético pelo método convencional

$\mathrm{O}$ valor energético foi determinado a partir da Equação de Atwater (Equação 1), considerando as médias dos valores obtidos para cada macronutriente a partir dos métodos convencionais.

\section{Determinação do teor de proteína}

O teor de proteína foi determinado pela quantificação de nitrogênio total da amostra, utilizando-se o destilador MicroKjeldhal (Tecnal, modelo TE-036/1). O teor de nitrogênio foi convertido em teor de proteína, multiplicando-se o valor encontrado pelo fator 6,25 . $^{7}$

\section{Determinação do teor de gordura}

Para a quantificação do teor de gordura das amostras foi usado o método de Bligh-Dyer, utilizando sulfato de sódio anidro (Vetec ${ }^{\circledR}$ ) para remover possíveis traços de água que tivessem permanecido na fase lipídica.

\section{Determinação do teor de umidade}

As análises de umidade foram realizadas por calor seco. As amostras com baixa quantidade de açúcares foram levadas em estufa de circulação de ar (Nova Ética, modelo 400/3ND) com temperatura de $105^{\circ} \mathrm{C}$ até peso constante. Já as demais amostras foram colocadas em estufa a vácuo (Tecnal, TE 395), a $60{ }^{\circ} \mathrm{C}$, para evitar a caramelização das mesmas.

\section{Determinação do teor de cinza}

Para a quantificação do teor de cinza, as amostras foram incineradas em mufla (Fornitec Rebert Shaw Pyrotec.), a $550{ }^{\circ} \mathrm{C}$, e retiradas após toda parte orgânica ter sido carbonizada (coloração branca a cinza). Depois, foram colocadas em dessecador para esfriar, sendo pesadas logo em seguida, em balança analítica (Ohaus, modelo Analytical Plus).

\section{Determinação do teor de carboidrato}

$\mathrm{O}$ teor de carboidrato foi calculado pela diferença entre o total da amostra (100\%) e os teores de proteína, gordura, umidade e cinza obtidos conforme descritos anteriormente.

\section{Determinação do valor energético pelo método proposto}

Para a obtenção das medidas por FRX, foram utilizadas celas Chemplex 1300, sustentadas por filme de Mylar $^{\circledR}$ (Chemplex 100) com 2,5 $\mu \mathrm{m}$ de espessura. As amostras foram transferidas para as celas até total preenchimento das mesmas. Cada amostra foi irradiada em triplicata, resultando em 300 espectros.

$\mathrm{O}$ espectrômetro utilizado foi de fluorescência de raios $\mathrm{X}$ por dispersão de energia (EDXRF), marca Shimadzu, modelo EDX-700, constituído por um tubo de Rh e um detector semicondutor de $\mathrm{Si}(\mathrm{Li})$. 
A voltagem aplicada no tubo de raios $\mathrm{X}$ foi de $50 \mathrm{kV}$, com $25 \%$ de tempo morto do detector. Os espectros foram obtidos sequencialmente, com uma resolução de $0,02 \mathrm{keV}$, de 0 a $40 \mathrm{keV}$. O tempo de irradiação foi de $120 \mathrm{~s}$.

Como valores de referência foram consideradas as médias dos resultados de VE obtidos pelo método convencional.

\section{RESULTADOS E DISCUSSÃO}

O tratamento dos dados foi realizado com os programas computacionais Pirouette $^{\circledR} 3.11$ (Infometrix Co., 2003) e Matlab ${ }^{\mathrm{TM}}$ versão 6.5 (Eigenvector).

Através dos espectros de fluorescência de raios X obtidos para as amostras, não foi possível distinguir ou agrupar as amostras conforme suas características (farinha ou cereal matinal, cereal presente, textura inicial da amostra, coloração etc.). Ou seja, usando um método univariado, não é possível verificar se o conjunto amostral apresenta alguma separação.

Na Figura 1 estão apresentados os espectros de 10 amostras $(10,20,30,40,50,60,70,80,90,100)$, nos quais se observa que os sinais mais intensos estão na região do espalhamento do raio $\mathrm{X}$ (18 e $24 \mathrm{keV})$.

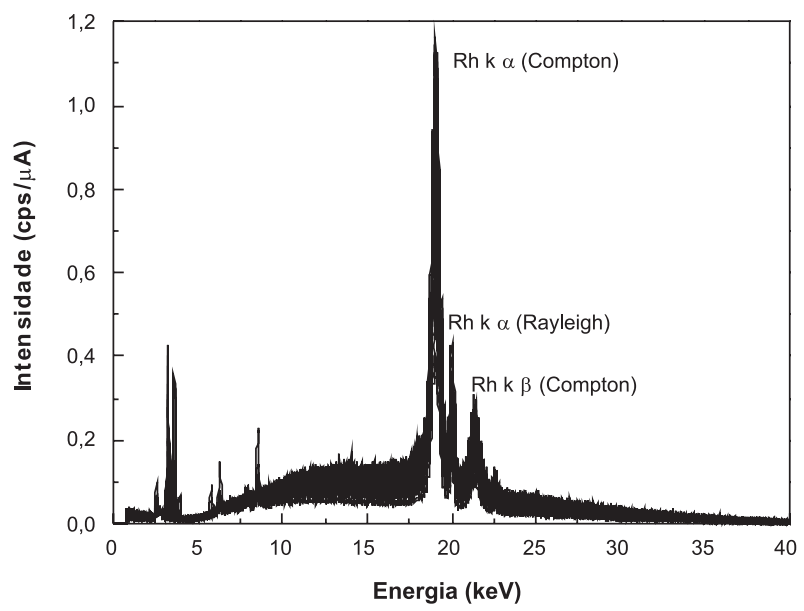

Figura 1. Espectros médios sobrepostos das amostras analisadas

Sendo assim, os espectros foram submetidos a tratamento quimiométrico por PCA para verificar a possibilidade de agrupamento das amostras; assim como PLS, para a construção dos modelos de calibração e validação de quantificação de VE.

Em todos os casos, o único pré-processamento utilizado foi centrar os dados na média, para facilitar a visualização dos dados. Este préprocessamento implica na subtração do elemento de cada coluna pelo valor médio dos elementos dessa coluna, obtendo-se como resultado uma matriz na qual todas as colunas têm média zero, ou seja, o centro do conjunto de dados fica na origem do sistema de eixos das componentes principais. ${ }^{28,29}$ Os espectros sofreram alisamento pelo método da média móvel, usando janela de 25 pontos, para aumentar a relação sinal/ruído.

A aplicação da PCA permitiu identificar que havia amostras anômalas no conjunto amostral. Isto foi comprovado pelo gráfico de leverage versus resíduos de Student. A leverage é uma medida de influência de uma amostra no modelo. Um valor de leverage pequeno indica que a amostra em questão influencia pouco na construção do modelo de calibração.

O resíduo de Student está relacionado ao resíduo da variável dependente (propriedade conhecida). Amostras mal modeladas terão resíduos altos. Supondo-se que os resíduos são normalmente distribuídos, e sabendo-se que eles são definidos em unidades de desvio padrão do valor médio, os valores com $\pm 2,5$ são considerados altos, sob as condições usuais de estatísticas. ${ }^{21}$

Todas as replicatas das amostras 18, 22, 25, 35 e 100, além das replicatas a da amostra 41 , b das amostras 38 e 40 e $\mathbf{c}$ das amostras 98 e 99 apresentavam altos valores tanto da leverage quanto do resíduo de Student e, portanto, foram excluídas do conjunto amostral.

As amostras excluídas não apresentavam nenhuma característica exclusiva e comum a elas que permitisse afirmar que o método proposto só pode ser aplicado para determinadas amostras de farinhas e cereais matinais. Alguns fatores podem ter sido responsáveis pelos altos valores de leverage e do resíduo de Student; entre eles, contaminação da cela de FRX e ranhuras na superfície do Mylar ${ }^{\circledR}$.

Após a exclusão das amostras anômalas, uma nova PCA foi realizada e não foram verificadas mais amostras que precisassem ser retiradas do conjunto por serem outliers (Figura 2). O modelo por PLS foi construído contendo todas as demais amostras.

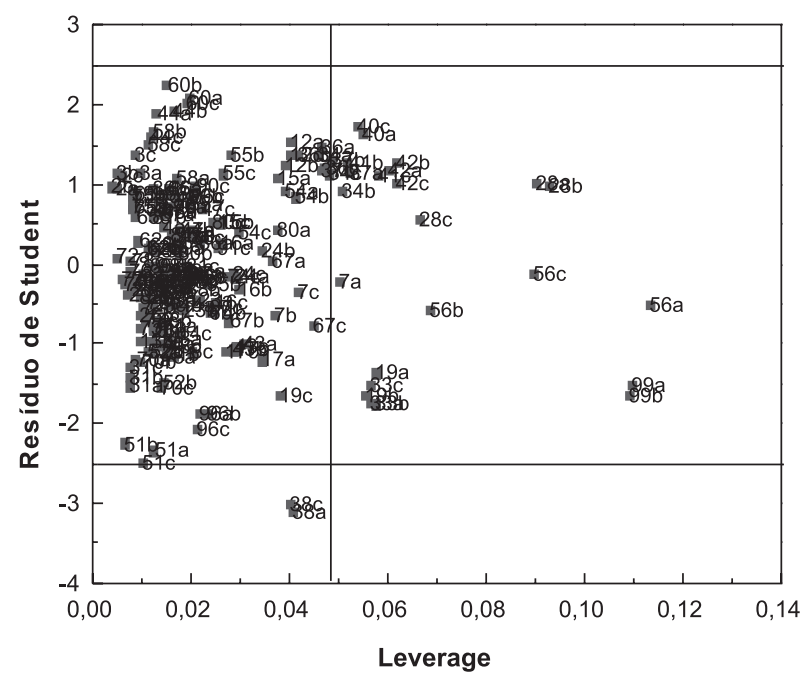

Figura 2. Representação gráfica da relação entre Resíduo de Student e Leverage para VE, após exclusão dos outliers, para 5 variáveis latentes

Este método quimiométrico permitiu estabelecer uma relação quantitativa entre os espectros de FRX e o valor energético das amostras, desenvolvendo um modelo matemático que correlaciona estas informações. O procedimento engloba duas etapas: calibração e validação. A etapa de calibração estabelece uma relação entre a matriz inicial de dados X (sinais instrumentais, variáveis independentes) e as propriedades conhecidas (de referência) das amostras (variáveis dependentes) organizadas na matriz $\mathrm{Y}^{30}$

Depois da calibração, é necessário realizar a validação do modelo para verificar se os erros de previsão são aceitáveis dentro do proposto. Um dos métodos mais utilizados para isto é a validação cruzada, na qual uma amostra é retirada do conjunto de $\mathrm{n}$ amostras e então é feita a calibração para as n-1 amostras restantes (variante leave-one-out). Na sequência, a amostra não usada na calibração tem sua variável prevista pelo modelo. O processo é repetido até que todas as amostras tenham sido testadas. ${ }^{24,31}$

Para verificar a capacidade de previsão do modelo para amostras que não pertenciam ao conjunto inicial é feita a validação externa, o que aumenta o grau de confiabilidade no modelo produzido. Os valores previstos para as novas amostras (amostras externas) são comparados com os valores de referência. ${ }^{23}$

Para a validação externa foram selecionadas aleatoriamente todas as replicatas de 24 amostras entre as não excluídas. As outras 71 amostras (211 espectros) foram utilizadas para realizar a calibração e a validação interna do método proposto. 
A escolha do número de variáveis latentes (LV) do modelo foi feita a partir do menor valor de PRESS (Predict Residual Error Sum of Squares). Este valor corresponde à soma dos quadrados dos erros de previsão e é calculado para cada $L V$, isto é, as novas variáveis resultantes das combinações realizadas para as variáveis originais. ${ }^{24,32}$

O modelo final foi feito com cinco variáveis latentes. Os loadings expressam o quanto cada uma das variáveis contribuiu para a formação do novo sistema de eixos, conforme mostra a Figura 3. Para este caso, foram importantes os elementos potássio $(3,3 \mathrm{keV})$, cálcio $(3,7 \mathrm{keV})$, manganês $(5,8 \mathrm{keV})$, ferro $(6,3 \mathrm{keV})$, zinco $(8,5$ $\mathrm{keV})$ e estrôncio (14 keV); o que pode ser verificado pelos valores significativos de loadings nas 5 primeiras LV (Figura 3). Os valores significativos de loadings entre 18 e $24 \mathrm{keV}$ permitem afirmar que as variáveis desta região também tiveram importância para o modelo, fato esperado uma vez que as amostras apresentam espécies orgânicas em sua composição (elementos leves).

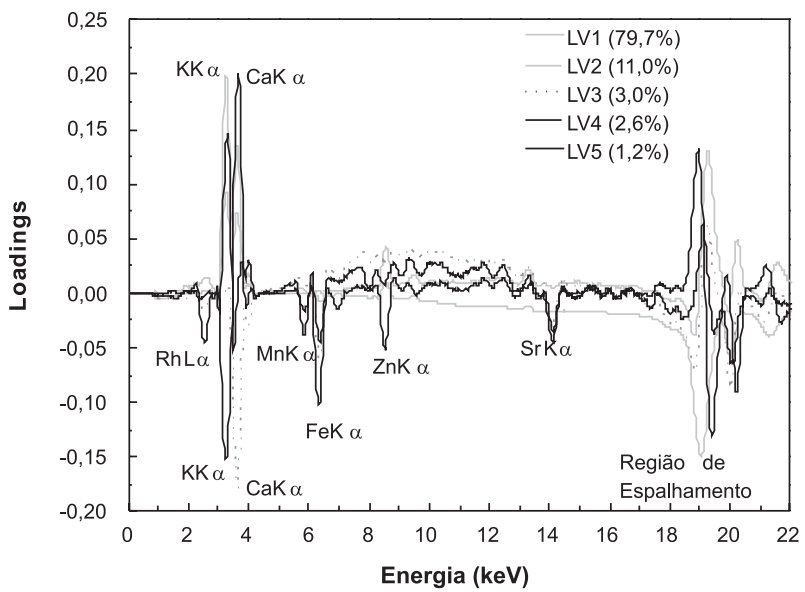

Figura 3. Gráfico de loadings das 5 primeiras LV para o modelo construído para $V E$, mostrando as variâncias explicadas em cada uma das variáveis latentes

Os valores médios de VE obtidos para as amostras a partir do modelo criado estão apresentados na Tabela 1 , onde também estão expostos os valores médios de referência (medidos pelo método convencional) e os respectivos erros entre tais valores.

A ANVISA (Agência Nacional de Vigilância Sanitária) é o órgão brasileiro que, entre outras funções, é responsável por "promover a proteção da saúde da população por intermédio do controle sanitário da produção e da comercialização de produtos e serviços submetidos à vigilância sanitária, inclusive dos ambientes, dos processos, dos insumos e das tecnologias a eles relacionados". ${ }^{2}$ É a ANVISA, portanto, que estabelece a legislação relacionada à elaboração dos rótulos dos alimentos embalados, além de fiscalizar o cumprimento das mesmas. Conforme a Resolução RDC 360, da Portaria 42, de 23 de dezembro de 2003, aos valores de nutrientes declarados no rótulo há uma tolerância de $20 \%{ }^{2}$

Observa-se na Tabela 1 que o maior erro da previsão interna foi de $15 \%$ (amostra 38), inferior ao permitido pela ANVISA.

Para verificar a adequação dos modelos via PLS foi usado o cálculo de algumas figuras de mérito conforme proposto por Poppi e colaboradores. ${ }^{25}$ Estes valores estão apresentados na Tabela 2.

O RMSEP (Root Mean Squares Error of Prediction) é similar ao desvio padrão e, portanto, sua unidade de medida é a mesma do valor energético, o parâmetro avaliado. Então, o RMSEP representa uma faixa de erro entre 4 e $6 \%$, uma vez que os valores obtidos pelo método convencional para esse parâmetro variavam entre 328 e 485 $\mathrm{kcal} / 100 \mathrm{~g}$. Nenhum dos valores medidos está abaixo dos valores dos limites de quantificação (LOQ) ou detecção (LOD), mostrando que o método é adequado para a aplicação proposta.
Tabela 1. Valores médios de referência e previstos pelo modelo proposto para VE, em kcal/100 g e erro percentual, em módulo, entre estes valores na validação interna.

\begin{tabular}{|c|c|c|c|c|c|c|c|}
\hline Amostra & $\begin{array}{c}\text { Valor de } \\
\text { Referência }\end{array}$ & $\begin{array}{c}\text { Valor } \\
\text { Previsto }\end{array}$ & |Erro| & Amostra & $\begin{array}{c}\text { Valor de } \\
\text { Referência }\end{array}$ & $\begin{array}{c}\text { Valor } \\
\text { Previsto }\end{array}$ & |Erro| \\
\hline 2 & 408 & 387 & 5 & 54 & 359 & 344 & 4 \\
\hline 3 & 393 & 367 & 7 & 55 & 372 & 349 & 6 \\
\hline 4 & 387 & 379 & 2 & 56 & 382 & 390 & 2 \\
\hline 6 & 374 & 382 & 2 & 57 & 437 & 425 & 3 \\
\hline 7 & 356 & 364 & 2 & 58 & 451 & 421 & 7 \\
\hline 8 & 356 & 377 & 6 & 59 & 423 & 409 & 3 \\
\hline 12 & 363 & 334 & 8 & 60 & 464 & 419 & 10 \\
\hline 13 & 353 & 371 & 5 & 62 & 413 & 408 & 1 \\
\hline 14 & 355 & 369 & 4 & 65 & 416 & 401 & 4 \\
\hline 15 & 398 & 383 & 4 & 66 & 388 & 368 & 5 \\
\hline 16 & 356 & 360 & 1 & 67 & 393 & 403 & 2 \\
\hline 17 & 364 & 387 & 6 & 68 & 401 & 394 & 2 \\
\hline 19 & 356 & 388 & 8 & 70 & 368 & 396 & 7 \\
\hline 20 & 366 & 373 & 2 & 71 & 377 & 396 & 5 \\
\hline 23 & 360 & 371 & 3 & 72 & 396 & 401 & 1 \\
\hline 24 & 348 & 349 & 0 & 73 & 399 & 411 & 3 \\
\hline 26 & 355 & 368 & 4 & 74 & 390 & 402 & 3 \\
\hline 28 & 363 & 346 & 5 & 75 & 409 & 393 & 4 \\
\hline 30 & 367 & 371 & 1 & 76 & 410 & 416 & 1 \\
\hline 31 & 354 & 383 & 8 & 78 & 401 & 403 & 1 \\
\hline 33 & 423 & 457 & 8 & 79 & 396 & 398 & 1 \\
\hline 34 & 475 & 451 & 5 & 80 & 394 & 387 & 2 \\
\hline 36 & 470 & 442 & 6 & 81 & 414 & 408 & 2 \\
\hline 37 & 467 & 442 & 5 & 82 & 396 & 401 & 1 \\
\hline 38 & 360 & 423 & 15 & 85 & 413 & 417 & 1 \\
\hline 40 & 485 & 450 & 7 & 86 & 430 & 419 & 3 \\
\hline 41 & 472 & 447 & 5 & 88 & 389 & 393 & 1 \\
\hline 42 & 467 & 443 & 5 & 89 & 390 & 397 & 2 \\
\hline 43 & 369 & 390 & 5 & 90 & 397 & 379 & 5 \\
\hline 44 & 409 & 370 & 9 & 91 & 388 & 389 & 0 \\
\hline 45 & 389 & 372 & 4 & 95 & 414 & 414 & 0 \\
\hline 46 & 361 & 363 & 1 & 96 & 391 & 432 & 9 \\
\hline 47 & 371 & 361 & 3 & 97 & 413 & 418 & 1 \\
\hline 48 & 355 & 373 & 5 & 98 & 413 & 414 & 0 \\
\hline 51 & 328 & 378 & 13 & 99 & 462 & 494 & 6 \\
\hline 52 & 352 & 377 & 7 & & & & \\
\hline
\end{tabular}

Tabela 2. Figuras de mérito do modelo multivariado obtidos para VE

\begin{tabular}{lccc}
\hline $\begin{array}{l}\text { RMSEP } \\
(\mathrm{kcal} / 100 \mathrm{~g})\end{array}$ & $\begin{array}{c}\text { LOQ } \\
(\mathrm{kcal} / 100 \mathrm{~g})\end{array}$ & $\begin{array}{c}\text { LOD } \\
(\mathrm{kcal} / 100 \mathrm{~g})\end{array}$ & $\mathrm{t}_{\text {bias }}$ \\
\hline 21 & 9 & 3 & 0,11 \\
\hline
\end{tabular}

O teste t permitiu concluir que os erros sistemáticos presentes no modelo são insignificantes, podendo ser desprezados, já que o $\mathrm{t}_{\text {bias }}$ calculado foi inferior ao $\mathrm{t}_{\text {Tabelado }}(1,96)$, com $95 \%$ de confiança.

Para verificar o poder de previsão do modelo para amostras externas, foram selecionados, aleatoriamente, cerca de $25 \%$ dos espectros não identificados como sendo de amostras anômalas (280). Desta forma, o conjunto de amostras externas envolvia um grupo de 72 espectros, correspondentes a 24 amostras. Na Tabela 3, estão apresentados os resultados médios obtidos no modelo de validação externa assim como os valores médios de VE calculados pelo método convencional e os erros entre estes valores. 
Tabela 3. Valores médios de referência e previstos pelo modelo proposto para VE, em kcal/100 g e erro percentual, em módulo, entre estes valores na validação externa

\begin{tabular}{cccccccc}
\hline Amostra & $\begin{array}{c}\text { Valor de } \\
\text { Referência }\end{array}$ & $\begin{array}{c}\text { Valor } \\
\text { Previsto }\end{array}$ & $\mid$ Erro $\mid$ & Amostra & \multicolumn{3}{c}{ Valor de } \\
Referência & \multicolumn{2}{c}{ Palor } & Previsto & Erro| \\
\hline 1 & 386 & 363 & 6 & 53 & 347 & 364 & 5 \\
5 & 381 & 384 & 1 & 61 & 394 & 399 & 1 \\
9 & 365 & 365 & 0 & 63 & 398 & 394 & 1 \\
10 & 371 & 360 & 3 & 64 & 397 & 409 & 3 \\
11 & 359 & 372 & 3 & 69 & 393 & 394 & 0 \\
21 & 356 & 373 & 5 & 77 & 393 & 390 & 1 \\
27 & 350 & 376 & 7 & 83 & 394 & 396 & 0 \\
29 & 350 & 362 & 3 & 84 & 395 & 388 & 2 \\
32 & 446 & 453 & 1 & 87 & 406 & 411 & 1 \\
39 & 475 & 455 & 4 & 92 & 409 & 403 & 1 \\
49 & 356 & 381 & 7 & 93 & 399 & 408 & 2 \\
50 & 348 & 356 & 2 & 94 & 397 & 410 & 3 \\
\hline
\end{tabular}

O maior erro de previsão para as amostras externas foi de $7 \%$ (amostra 27), bem menor do que os $20 \%$ aceitos pela ANVISA; o que permite concluir que ao modelo podem ser incluídas amostras com valores de referência desconhecidos para serem previstos dentro da faixa de erro atual exigida pelos órgãos competentes.

Como curiosidade, foram comparados os valores obtidos para valor energético pelo método convencional e os valores apresentados no rótulo (Tabela 4). Esta comparação permitiu verificar que 8 das 100 amostras analisadas (8\%) apresentaram erros superiores a $20 \%$. Isso deve refletir que as indústrias alimentícias estão cumprindo as normas das ANVISA, pelo menos para VE, possivelmente por ser esta uma das informações de maior apelo comercial na busca de consumidores.

\section{CONCLUSÕES}

Os resultados permitiram verificar a potencialidade da aplicação da técnica de fluorescência de raios $\mathrm{X}$ aliada à quimiometria para se determinar o valor energético em amostras de farinhas e cereais matinais para consumo humano.

O tempo reduzido de análise ( $0,2 \%$ do tempo necessário para a aplicação do método convencional) e a não necessidade de uso de solventes tornam o método proposto uma alternativa a ser considerada em substituição ao método convencional de análise empregado atualmente, principalmente em laboratórios industriais e de rotina. Além disso, os erros de previsão externa foram inferiores ao permitido atualmente (20\%) pela Agência Nacional de Vigilância Sanitária, o que reforça a aplicação da proposta.

\section{REFERÊNCIAS}

1. Celeste, R. K.; Rev. Saúde Pública 2001, 35, 217.

2. BRASIL, Resolução da diretoria colegiada 360 de 23 de dezembro de 2003; Regulamento Técnico sobre Rotulagem Nutricional de Alimentos Embalados Diário Oficial da República Federativa do Brasil, Brasília, DF, 26/12/2003, Seção 1, p. 28.

3. Kays, S. E.; Windham, W. R.; Barton II, F. E.; J. Agric. Food Chem. 1996, 44, 2266.

4. Agarwal, S.; Hordvik, S.; Morar, S.; Toxicology 2006, 21, 44.

5. Torres, E. A. F. S.; Campos, N. C.; Duarte, M.; Garbelotti, M. L.; Phillipi, S. T.; Minazzi-Rodrigues, R. S.; Ciênc. Tecnol. Aliment. 2000, 20, 145.

6. Atwater, W. O.; Bryant, A. P.; $12^{\text {th }}$ Annual Report (1899) of the Storrs, CT Agricultural Experimental Station, University of Connecticut, Storrs: CT, 1900, p. 73-110.
Tabela 4. Valores médios de referência e valor de rótulo para VE, em kcal/100 g e erro percentual, em módulo, entre estes valores

\begin{tabular}{|c|c|c|c|c|c|c|c|}
\hline Amostra & $\begin{array}{c}\text { Valor de } \\
\text { Referência }\end{array}$ & $\begin{array}{l}\text { Valor de } \\
\text { Rótulo }\end{array}$ & |Erro| & Amostra & $\begin{array}{l}\text { Valor de } \\
\text { Referência }\end{array}$ & $\begin{array}{l}\text { Valor de } \\
\text { Rótulo }\end{array}$ & |Erro| \\
\hline 1 & 386 & 332 & 14 & 51 & 328 & 360 & 9 \\
\hline 2 & 408 & 410 & 0 & 52 & 352 & 355 & 1 \\
\hline 3 & 393 & 600 & 34 & 53 & 347 & 345 & 1 \\
\hline 4 & 387 & 433 & 11 & 54 & 359 & 338 & 6 \\
\hline 5 & 381 & 393 & 3 & 55 & 372 & 380 & 2 \\
\hline 6 & 374 & 397 & 6 & 56 & 382 & 319 & 17 \\
\hline 7 & 356 & 358 & 1 & 57 & 437 & 408 & 7 \\
\hline 8 & 356 & 360 & 1 & 58 & 451 & 400 & 11 \\
\hline 9 & 365 & 370 & 1 & 59 & 423 & 415 & 2 \\
\hline 10 & 371 & 370 & 0 & 60 & 464 & 398 & 14 \\
\hline 11 & 359 & 340 & 5 & 61 & 394 & 393 & 0 \\
\hline 12 & 363 & 373 & 3 & 62 & 413 & 372 & 10 \\
\hline 13 & 353 & 360 & 2 & 63 & 398 & 348 & 13 \\
\hline 14 & 355 & 360 & 1 & 64 & 397 & 260 & 35 \\
\hline 15 & 398 & 340 & 15 & 65 & 416 & 345 & 17 \\
\hline 16 & 356 & 344 & 3 & 66 & 388 & 360 & 7 \\
\hline 17 & 364 & 180 & 51 & 67 & 393 & 360 & 9 \\
\hline 18 & 356 & 150 & 58 & 68 & 401 & 369 & 8 \\
\hline 19 & 356 & 150 & 58 & 69 & 393 & 380 & 3 \\
\hline 20 & 366 & 340 & 7 & 70 & 368 & 300 & 19 \\
\hline 21 & 356 & 340 & 5 & 71 & 377 & 367 & 3 \\
\hline 22 & 346 & 327 & 5 & 72 & 396 & 337 & 15 \\
\hline 23 & 360 & 338 & 6 & 73 & 399 & 215 & 46 \\
\hline 24 & 348 & 350 & 1 & 74 & 390 & 350 & 10 \\
\hline 25 & 355 & 350 & 1 & 75 & 409 & 387 & 5 \\
\hline 26 & 355 & 350 & 1 & 76 & 410 & 400 & 2 \\
\hline 27 & 350 & 340 & 3 & 77 & 393 & 370 & 6 \\
\hline 28 & 363 & 381 & 5 & 78 & 401 & 380 & 5 \\
\hline 29 & 350 & 350 & 0 & 79 & 396 & 373 & 6 \\
\hline 30 & 367 & 365 & 0 & 80 & 394 & 373 & 5 \\
\hline 31 & 354 & 400 & 12 & 81 & 414 & 403 & 3 \\
\hline 32 & 446 & 413 & 7 & 82 & 396 & 380 & 4 \\
\hline 33 & 423 & 442 & 4 & 83 & 394 & 373 & 5 \\
\hline 34 & 475 & 490 & 3 & 84 & 395 & 373 & 5 \\
\hline 35 & 329 & 355 & 7 & 85 & 413 & 350 & 15 \\
\hline 36 & 470 & 500 & 6 & 86 & 430 & 425 & 1 \\
\hline 37 & 467 & 469 & 0 & 87 & 406 & 348 & 14 \\
\hline 38 & 360 & 200 & 44 & 88 & 389 & 381 & 2 \\
\hline 39 & 475 & 460 & 3 & 89 & 390 & 376 & 4 \\
\hline 40 & 485 & 433 & 11 & 90 & 397 & 376 & 5 \\
\hline 41 & 472 & 460 & 3 & 91 & 388 & 376 & 3 \\
\hline 42 & 467 & 500 & 7 & 92 & 409 & 397 & 3 \\
\hline 43 & 369 & 200 & 46 & 93 & 399 & 398 & 0 \\
\hline 44 & 409 & 357 & 13 & 94 & 397 & 397 & 0 \\
\hline 45 & 389 & 357 & 8 & 95 & 414 & 408 & 1 \\
\hline 46 & 361 & 356 & 1 & 96 & 391 & 381 & 3 \\
\hline 47 & 371 & 380 & 2 & 97 & 413 & 364 & 12 \\
\hline 48 & 355 & 358 & 1 & 98 & 413 & 400 & 3 \\
\hline 49 & 356 & 350 & 2 & 99 & 462 & 467 & 1 \\
\hline 50 & 348 & 355 & 2 & 100 & 400 & 400 & 0 \\
\hline
\end{tabular}

7. Buchholz, A. C.; Schoeller, D. A.; Am. J. Clin. Nutr. 2004, 79, 899.

8. AOAC International; AOAC International Official Methods of Analysis, $16^{\text {th }}$ ed., AOAC International: Gaithersburg, 1996. 
9. Bligh, E. C.; Dyer, W. J.; Can. J. Biochem. Physiol. 1959, 37, 911.

10. Aguilera, J. M.; Stanley, D. W.; Microstructural Principles of Food \& Engineering, $1^{\text {st }}$ ed., Elsevier: Cambridge, 1990.

11. Bobbio, P. A.; Bobbio, F. O.; Química do processamento de alimentos, Varela: São Paulo, 1992.

12. Cecchi, H. M.; Fundamentos Teóricos e Práticos em Análise de Alimentos, Ed. UNICAMP: Campinas, 1999.

13. Fennema, O. R.; Food Chemistry, $3^{\text {rd }}$ ed., Marcel Dekker: New York, 1996.

14. Prentice, A. M.; Public Health Nutr. 2005, 8, 932.

15. Takeuchi, K. P.; Sabadini, E.; Cunha, R. S.; Ciênc. Tecnol. Aliment. $\mathbf{2 0 0 5}, 25,78$.

16. http://old.iupac.org/publications/compendium/X.html, acessada em Abril 2010.

17. Jenkins, R.; De Vries, J. L.; Practical X-Ray Spectrometry, $2^{\text {nd }}$ ed., Springer: New York, 1970.

18. Skoog, D. A.; Holler, F. J.; Nilman, T. A.; Princípios da Análise Instrumental, $5^{\text {a }}$ ed., Bookman: Porto Alegre, 2002.

19. Bortoleto, G. G.; Borges, S. S. O.; Bueno, M. I. M. S.; Anal. Chem. Acta 2007, 595, 38.

20. Pereira, F. M. V.; Bueno, M. I. M. S.; Chem. Intell. Lab. Syst. 2008, 92 , 131.
21. Ferreira, M. M. C.; Antunes, A. M.; Melgo, M. S.; Volpe, P. L. O.; Quim. Nova 1999, 22, 724 .

22. Moita Neto, J. M.; Moita, G. C.; Quim. Nova 1998, 21, 467.

23. Martens, H.; Naes, T.; Multivariate Calibration, Wiley: New York, 1996.

24. Beebe, K. R.; Pell, R. J.; Seasholtz, M. B.; Chemometrics: A Practical Guide, John Wiley \& Sons: New York, 1998.

25. Valderrama, P.; Braga, J. W. B.; Poppi, R. J.; J. Braz. Chem. Soc. 2007, $18,259$.

26. Baccan, N.; Andrade, J. C.; Godinho, O. E. S.; Barone, J. S.; Química Analítica Quantitativa Elementar, $3^{\text {a }}$ ed., Edgard Blücher: São Paulo, 2001.

27. Miller, J. N.; Miller, J. C.; Statistics and Chemometrics for Analytical Chemistry, Pearson Education: Harlow, 2000.

28. Otto, M.; Chemometrics - Statistics and Computer application in Analytical Chemistry, Wiley: Weinheim, 1999.

29. Thomas, E. V.; Anal. Chem.1994, 66, 795.

30. Geladi, P.; Kowalski, B. R.; Anal. Chim. Acta 1986, 185, 7.

31. Vandeginste, B. G. M.; Massart, D. L.; Buydens, L. M. C.; De Jong, S.; Lewi, P. J.; Smeyers-Verbeke, J.; Handbook of Chemometrics and Qualimetrics: Part B, $1^{\text {st }}$ ed., Elsevier: Amsterdam, 1998.

32. Sharaf, M. A.; Liman, D. L.; Kowalski, B. R.; Chemometrics, Wiley: New York, 1986. 